\title{
THE FOUNDATIONAL CONCEPTS OF CLINICAL PRACTICE
}

\author{
Nancy C. Tkacs, Linda L. Herrmann, Randall L. Johnson, and Loretta A. Sernekos
}

\section{PATHOPHYSIOLOGY AND THE PROCESS OF CLINICAL DECISION-MAKING}

Clinicians' ability to correctly identify disease processes and develop management plans with their patients relies on a deep knowledge of the pathophysiology of disease. This book was developed to meet that clinical need. Physiology seeks to understand the mechanisms of body function, and pathophysiology seeks to understand the mechanisms of altered body function due to pathological states. People, whether patients or clinicians, often fail to appreciate the finetuned machine that is the human body until they experience alterations in function that lead to signs and symptoms of disease. Pathophysiology can be viewed with the same lens: Studying the manifestations of body function loss can illuminate the exquisite and intricate functions of our organs that we do not even think about when we are healthy. For every physiological function, there is a pathophysiological consequence when that normal function goes awry. Clinicians-in-training are well positioned to reinforce their knowledge of normal physiological principles when they are presented as the context for disease development, as we have in this book.

In disease states, one or more normal homeostatic mechanisms malfunction, and the organism increasingly relies on compensatory mechanisms to maintain homeostasis. The clinician must know the principles of normal function, the alteration of these functions during disease states, and the benefits and risks of compensatory mechanisms. In addition, one cannot study physiology and pathophysiology in isolation: To understand the complex mechanisms of body function requires application of fundamental principles from chemistry, genetics, cell biology, and other basic sciences.

The data gathered during the clinical encounter include details from the history and physical examination, comprising both subjective and objective findings; results of laboratory studies; results of imaging studies; and other measurements. The clinician must then evaluate these data, consider the differential diagnosis, and formulate a plan. At each step, knowledge of pathophysiology is integral to the process. The aims of this book are to:

- Provide a foundation of core principles of physiology for each system and organ discussed

- Link the pathophysiology of common and selected less common disorders to those core principles

- Alternate discussion of each core principle with the clinical applications, sequencing the content to emphasize the application of those core principles and mechanisms

- Present in-depth life span considerations for each organ and system as developed by geriatric and pediatric content experts who

- Emphasize developmental aspects of organ functions and their relationships to disorders commonly encountered in infants and children

- Describe organ and system alterations of normal healthy aging and their relationships to disorders commonly encountered in older adults

- Provide a bridge between pathophysiology and concurrent or later coursework on health history and physical assessment, diagnostic tools and laboratory testing, pharmacology, and nonpharmacologic management strategies 
- Offer an interdisciplinary perspective on the intricacies of the body's systems in both a normal and a diseased state, with an author team comprising experienced clinicians and educators: nurses and nurse practitioners, physician assistants, dietitians, doctors of pharmacy, physicians, and basic scientists

At the same time, we endeavored to avoid redundancy with topics covered more extensively in other courses in the graduate curriculum of advanced practitioners. The book does not have a freestanding chapter on the skin, for example. The most common skin disorders have unique presentations that are well described in physical assessment books and clinical lectures on dermatology. We feel that these disorders are best covered in textbooks associated with those topics. Rather than devoting a lengthy chapter to this topic, the mechanisms of selected skin disorders are covered within the context of infectious disease (Chapter 5, Infectious Disease) and immune and leukocyte function (Chapter 6, The Immune System and Leukocyte Function), which are the primary origins of many skin disorders and consistent with our approach of discussing disease in the context of alterations of existing physiological principles and mechanisms.

Similarly, we have selected certain common and uncommon disorders for discussion in the book because of their educational value and the way they illustrate an underlying principle of function. In many curricula, the pathophysiology course spans only one semester, making it impossible to describe an exhaustive list of diseases in detail. While this book explores a wide range of disorders, our approach aims to provide mastery of concepts, vocabulary, and diagnostic evaluation strategies that students can use throughout their remaining clinical semesters to broaden their knowledge of diseases they encounter. Each principlefocused segment ends with Thought Questions to immediately engage the students in reflection and analysis of their level of mastery of that chunk of content. Chapters conclude with Key Points to return students to the big picture of function and dysfunction for the chapter's main topics. We sincerely hope that this approach produces a resource that students will actually read and keep reading as a companion to their subsequent coursework.

\section{BUILDING FROM A BASIC SCIENCE FOUNDATION: CHAPTERS 2 TO 4}

Physiological and pathophysiological processes are carried out at the level of chemistry, biochemistry and biophysics, and molecular and cellular biology. Owing to the rapid pace of scientific discovery in these fields, our knowledge can never be complete, and independent practitioners must make a commitment to lifelong learning. A clear understanding of important concepts and terminology from these disciplines contributes to the ability of clinicians to continually update their knowledge as science advances.

Examples of clinical applications of these disciplines are highlighted here. They underscore the importance of the basic sciences for clinical decision-making, diagnosis, and management, as well as for understanding new research and clinical innovations. Chapters 2 to 4 provide brief reviews of chemistry, biochemistry, molecular biology and genetics, and cell biology that cover principles and terminology of these disciplines that are used in all subsequent chapters. These chapters could be the basis for a short online review course or for self-study to increase students' success and satisfaction in their graduate pathophysiology coursework.

\section{CHEMISTRY (CHAPTER 2)}

All body functions can ultimately be described in terms of chemical interactions. The body's chemistry begins at the level of single atoms, including many of the electrolytes. Sodium, chloride, potassium, calcium, and hydrogen ions must be kept within a normal range of concentration in both the intracellular and extracellular fluid. Ion imbalance can lead to cardiac arrhythmias, paresthesias, and acidosis. Minerals, including those present in small amounts (trace minerals), are required for proper function of many enzymes. Iron is the site of oxygen binding within hemoglobin and myoglobin molecules, and iodine is required for thyroid hormone synthesis. The chemistry of the body has an undisputed role in health and disease.

Atoms combine through ionic and covalent bonds to form molecules. In addition to the previously listed elements, key atoms that make up the molecules of the body are carbon, hydrogen, oxygen, nitrogen, phosphorus, and sulfur. Molecular oxygen $\left(\mathrm{O}_{2}\right)$ is needed by all cells for synthesis of adenosine triphosphate (ATP) and other energy sources. This role of oxygen comes with a cost, in that oxidative metabolism generates oxygenderived free radicals that can damage cell components.

The most abundant molecule in the body is water, and the polar nature of water's molecular structure is a major determinant of physiological function. The aqueous environment of the body's extracellular and intracellular fluids creates the shape and interactions of proteins, lipids, and carbohydrates to sustain life. The nonpolar, hydrophobic molecular nature of fatty acids underlies the function of the plasma membrane as a barrier between intracellular and extracellular fluids. With a hydrophobic fatty acid core, the plasma membrane defines the cell, the smallest unit of biological function.

Solubility, diffusion, equilibrium reactions, and many other core concepts of atomic and molecular function will appear throughout this book. 


\section{BIOCHEMISTRY (CHAPTER 2)}

The biomolecules-carbohydrates, lipids, proteins, and nucleic acids - are the workhorses of cell and organ function. Abnormalities in metabolism, such as phenylketonuria, result in abnormally high levels of certain biomolecules and abnormally low levels of others, transforming function in devastating ways. Nutritional deficiencies (iron-deficiency anemia) and excesses (obesity) underlie many disease processes and may be preventable or readily treatable, once recognized.

\section{MOLECULAR BIOLOGY AND GENETICS (CHAPTER 3)}

The human genome project was completed in 2003, and was followed by additional haplotype mapping studies to identify common variant alleles and singlenucleotide polymorphisms (SNPs). The impact of these developments on clinical practice is profound and far reaching. Understanding heredity, single gene disorders, penetrance, expressivity, and the impact of human gene variants on disease risk is integral to clinicians entering independent practice. Common genetic disorders and variants are discussed in this book, and each chapter features one or more highlighted genetic disorders. Some less common genetic disorders are included because they illustrate important concepts in genetic disease.

The consequences of many genetic disorders are detectable in the perinatal period, as described in the Pediatric Considerations section of this chapter. Prenatal genetic screening and diagnosis are discussed here, along with genetic disorders identified after birth. Newborn screening programs continually expand their targets-identifying genetic and biochemical abnormalities in enzymes and other biomolecules within a few days after birth, allowing early intervention and management that prevents or lessens long-term disability.

\section{CELL BIOLOGY (CHAPTER 4)}

As molecular biology knowledge has expanded, so too has knowledge of cell biology. Membrane transport proteins and membrane-bound receptors are the targets of many drug classes in common use, and new subtypes of these membrane proteins continue to be discovered. Knowledge of intracellular signaling cascades involved in immune function and neoplastic transformation is growing rapidly, leading to better tools for management of autoimmunity, hypersensitivity, and cancer.

A fundamental understanding of the impact of aging at cellular, tissue, organ, and systemic levels is crucial in order for clinicians to tailor individualized ageappropriate interventions, given the demographics on aging domestically and abroad. By 2060, 23\% of U.S. residents will be aged 65 and older, an increase from $16 \%$ in 2018. ${ }^{1}$ In 2015, the number of persons aged 60 and older worldwide was 900 million; this is expected to increase to 2 billion people by $2050 .^{2}$ Knowledge of the pathophysiological underpinnings of age-related changes, both normal and abnormal, is paramount when considering changes in implicit body regulatory mechanisms, metabolism, and nutrition; selecting pharmacotherapy; and critically analyzing the interplay of systemic manifestations of disease, clinical data, and patient presentation. Hypotheses of aging at the cellular level are described in the Gerontological Considerations section of Chapter 4.

\section{ADVANCED PHYSIOLOGY AND PATHOPHYSIOLOGY: CHAPTERS 5 TO 17}

After introducing the building blocks of physiological and pathophysiological function, the book transitions, first to an overview of infectious disease mechanisms with exemplar organisms, then to a system-by-system walkthrough of the body, reviewing principles of function and their clinical applications. An example of the book's sequencing approach is shown in Figure 1.1, which visually maps how foundational principles and concepts relating to the lung are linked with specific pulmonary disorders. Selected disorders receive an in-depth discussion, and learners are introduced to principles of dysfunction related to each mechanism that can be generalized to many other disorders. We believe that this approach best serves the short-term goal of providing knowledge of pathophysiology of specific diseases, and the long-term goal of mastering pharmacological and clinical applications. These clinically related chapters also feature specific sections on Pediatric and Gerontological Considerations, Case Studies, and a unique Bridge to Clinical Practice segment that briefly summarizes principles of assessment, diagnostic tools and laboratory evaluation, and major drug classes pertinent to the chapter's main topic.

\section{INFECTIOUS DISEASE (CHAPTER 5)}

In keeping with our emphasis on principles and concepts, this chapter begins with an overview of the interactions between humans and microbes, and concepts such as infection versus colonization, pathogen virulence, and host characteristics that determine infection severity. A brief review of characteristics of bacteria, viruses, protozoa, fungi, and yeasts is provided, followed by clinical concepts of pathogen identification strategies, drug resistance, and antimicrobial stewardship. This chapter concludes with snapshots of the structure and function of selected microbes commonly encountered in clinical settings in the United States or globally. Selected infectious diseases are further discussed in the relevant organ systems chapters; for example, pneumonia in Chapter 11, Lungs, and infectious diarrheas in Chapter 13, Gastrointestinal Tract. 
Advanced physiology and pathophysiology:

A sequenced approach to disease mechanisms

\section{Physiology and pathophysiology of the lung}

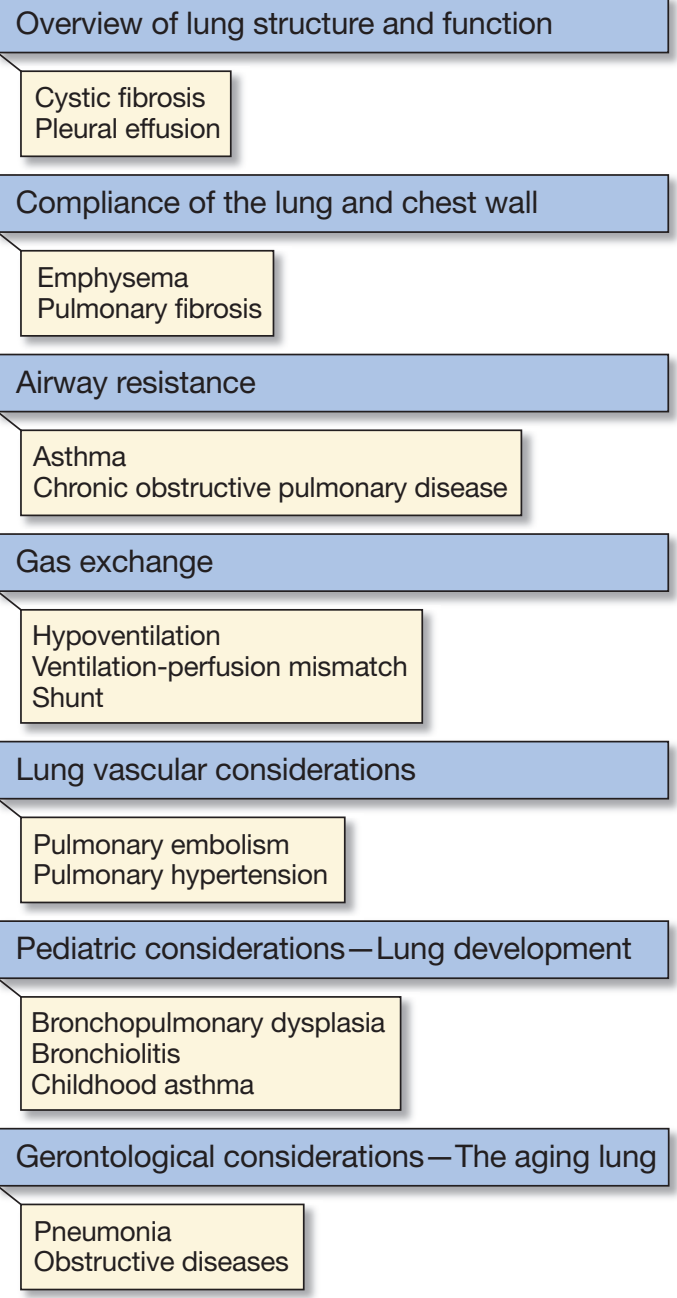

FIGURE 1.1 The organization and sequence of topics in Chapter 11, Lungs, focusing on the lungs, illustrate the overarching approach used throughout this book. Main principles and concepts in organ function (shown in blue boxes) are immediately followed by descriptions of disorders linked to pathophysiology of those principles and concepts (shown in beige boxes).

\section{THE IMMUNE SYSTEM AND LEUKOCYTE FUNCTION (CHAPTER 6)}

Knowledge of immunology continues to grow exponentially, and identification of lymphocyte subsets, cytokines, and other members of the immune system's "cast of characters" continues to provide new targets for biologic drugs. At the same time, innate immunity (inflammation) continues to cause a great deal of morbidity and mortality, wreaking havoc in the blood vessels of patients with atherosclerosis, the adipose tissue of persons with obesity and metabolic syndrome, and the joints of patients with rheumatoid arthritis. This chapter explores the latest concepts of innate and adaptive immunity; details the pathophysiology of hypersensitivity, autoimmunity, and immunodeficiency; and builds the foundation for understanding the pharmacology of biological therapies for immune-related disorders. The waxing and waning of immune function across the life span, and implications for care of both children and older adults, are placed in that context.

\section{NEOPLASIA (CHAPTER 7)}

This chapter focuses on the principles underlying a cell's movement into the cell cycle and cell divisionthe critical event that is inappropriately activated in cells that have undergone neoplastic transformation. Characteristics of cancer cells are described, with emphasis on the implications for current and projected treatments of certain cancers. The discussion of genetic and viral causes of cancer highlights the critical role of genotyping in cancer care. The most common cancers of children are described, as well as specific aspects of cancer pathophysiology in older adults.

\section{BLOOD AND CLOTTING (CHAPTER 8)}

This chapter builds on the content of Chapter 6 , The Immune System and Leukocyte Function, by focusing on red blood cells in health and disease and the processes of blood clotting. The concept of anemia and the principles underlying the different forms of anemia provide the foundation for developing differential diagnoses in patients with suspected anemia based on clinical manifestations and diagnostic findings. The process of hemostasis is described, focusing on the complementary roles of platelets and coagulation factors, as well as relevant laboratory assessments. This underpins the subsequent discussion of pathophysiological and genetic causes of states of excess clotting and excess bleeding. Life span considerations include the unique changes in red blood cell size and number in the perinatal period, and the bone marrow-related changes of older adults.

\section{CIRCULATION (CHAPTER 9)}

The circulatory system receives the output of the heart and channels the blood flow through a series of tubes that diverge and narrow as they proceed outward to the tissues, and then converge and enlarge as they return to the heart. Although some aspects of this flow are true for all segments of the circulation, structural and biophysical characteristics of each segment determine the pathophysiological vulnerabilities of each. The large muscular arteries move blood rapidly and efficiently 
to various body sites but are prone to atherosclerosis, as well as hypertrophy and aneurysm. The arterioles provide vascular resistance that helps to maintain organ perfusion pressures - they are the site of blood pressure control by neural and humoral mediators, and their dysfunction is associated with hypertension and shock. The capillaries exchange essential substances with the tissues, and are also the locus of physiological and pathophysiological edema formation. The veins are low-pressure storage vessels that are vulnerable to gravitational influences, varicosities, and coagulability. Hypertension can occur in children as well as adults, and recognizing this disorder depends on knowledge of age-related population norms. Older adults have additional clinical concerns related to vascular stiffness due to age-related collagen deposition in vessel walls.

\section{HEART (CHAPTER 10)}

The heart is an autonomously functioning pump, ejecting a volume of blood (stroke volume) with each beat into the circulation for delivery to the body. Heart function is critically dependent on electrical automaticity of pacemaker cells and rapid action potential propagation that produces coordinated chamber contractions. Cardiac cells have unique action potentials that can be vulnerable to abnormal cardiac beats and rhythms (arrhythmias). These electrical signals are linked to calcium entry that initiates the mechanical steps of contraction-the pumping action of the heart. The mechanical properties of the heart determine the stroke volume- the amount of blood pumped with each beat, at rest and during exercise, to meet the body's need for oxygen delivery and carbon dioxide removal. Ischemic heart disease results in both electrical and mechanical dysfunction. Recently, research has begun to illuminate how the pathophysiology and presentation of ischemic heart disease differ in men and women, and clinical practice is beginning to change accordingly.

Heart failure can result from ischemic heart disease, among other causes, and is a chronic, progressive loss of pumping capacity that ultimately limits physical activity and can result in death. Structural cardiac disorders range from valve diseases in children and adults to malformations arising during embryonic and fetal development. Many of these are managed surgically, albeit with varying degrees of success depending on severity. Cardiac changes during healthy aging generally do not result in activity limitations; however, many disorders, such as atrial fibrillation, show increased prevalence with aging.

\section{LUNGS (CHAPTER 11)}

The lungs interface with the environment, bringing in atmospheric air during inspiration, and expelling mixed airway and alveolar air during expiration. Thus, the airways - the diverging and narrowing tubes that lead to the alveoli-are exposed to pollutants, irritants, and pathogens. Several mechanisms, including antimicrobial and mucociliary responses, protect the airways from these damaging substances. The lungs' delicate structure is encased in a more rigid thoracic chamber, surrounded by the chest wall (ribs, sternum, associated muscles and lining), and bounded below by the diaphragm. The ability of inspiratory muscles (diaphragm and others) to inflate the lungs depends on compliance of the lungs and the chest wall, and pathophysiological changes in either component can alter the amount of effort required for inspiration. The airways are the site of airway resistance that must be overcome to generate inspiratory and expiratory airflow. Airway diameter can be pathologically altered in a variety of disease processes, leading to acute or chronic obstructive disease.

Gas exchange occurs in the alveoli and depends on lung mechanics (for ventilation) as well as blood flow (for perfusion) in taking up oxygen and releasing carbon dioxide. Diseases may alter blood oxygenation (and carbon dioxide removal) through their effects on ventilation, perfusion, or the diffusion barrier of the alveolar wall. The lungs receive all of the blood flow of the right heart, a low-pressure system that perfuses the millions of alveolar capillaries for gas exchange. Abnormalities of lung blood flow can lead to secondary heart disease, lung fluid accumulation, and acutely impaired oxygenation. Much of the alveolar development occurs postnatally, and preterm birth is associated with persistent lung pathology. In older adults, pneumonia is a common occurrence, related in part to decreased protective mechanisms.

\section{KIDNEYS (CHAPTER 12)}

The kidneys are highly vascular organs that filter the blood, removing wastes for excretion and returning the rest of the blood to the vascular system. The functional units of the kidneys are nephrons, each composed of a glomerulus and a tubule. In a two-step process, the glomeruli receive blood for filtration and release an ultrafiltrate of plasma into the tubules, which then process the filtrate and greatly reduce its volume, leading to the final step of urine production. The extremely high blood flow and capillary permeability of the glomeruli relative to other vascular beds make them vulnerable to damage by hypertension, diabetic hyperglycemia, antibodies, and complement components. The tubules are transport membranes that return most of the glomerular filtrate to the circulation, while secreting substances destined for clearance, including many drugs. Tubules can be damaged by many filtered and secreted substances, including proteins such as myoglobin and certain medications. Hypotension and hypovolemia can also lead to tubular necrosis in the renal medulla, which receives relatively low blood flow and is prone to hypoxia. Acute kidney injury may resolve, but 
compromised function may progress to chronic kidney injury and end-stage renal disease. At both ends of the life span, childhood and older age, the kidneys are more vulnerable to damage owing to decreased number or function of nephrons.

\section{GASTROINTESTINAL TRACT (CHAPTER 13)}

Similar to the circulatory system, the basic structure of the gastrointestinal tract is a generalized tube with a series of segments that are specialized in structure and function. Similar to the respiratory tract, the gut is exposed to environmental toxins-in this case, a wide variety of ingested foods and beverages, as well as pathogens. In addition to a sequential description of structure, function, and disorders of the esophagus, stomach, and small and large intestines, this chapter emphasizes general concepts of gut control by neurotransmitters and hormones that are the basis for many of the drugs used to manage gastrointestinal disorders. The abundant and intricate immune system of the gut plays a role in development of immune responses in early life and is protective against ingested pathogens, but also is implicated in inflammatory bowel disease. Life span considerations include structural disorders as well as common infectious diarrheas of children, and structural disorders of older adults, including greater occurrence of reflux and diverticular disease.

\section{LIVER (CHAPTER 14)}

This book places a substantial emphasis on the physiology and pathophysiology of the liver, providing a critical foundation for clinicians who will be responsible for prescribing medications. We have dedicated a chapter solely to this organ rather than including it in the gastrointestinal tract chapter because drug-induced liver injury is extremely common, and prescribers must appreciate the role of the liver in drug metabolism and excretion. Understanding the metabolic functions of the liver is also key to appreciating its significance in diabetes, the metabolic syndrome, and nonalcoholic fatty liver disease - a disorder with steadily increasing prevalence in the developed world. Infectious hepatitis is common in the United States and globally; an understanding of the patterns of dysfunction and laboratory assessment of different subtypes of hepatitis is critical for advanced practice. Individuals at both ends of the life span are more vulnerable to liver injury-children, because of the relative immaturity of many of the drug-metabolizing enzymes, and older adults, because of reduced liver mass and blood flow.

\section{NERVOUS SYSTEM (CHAPTER 15)}

The nervous system is arguably the most complex body system, with both physiological and behavioral components, all of which make up the whole person cared for by clinicians. To provide a strong foundation of knowledge for practitioners with prescriptive privileges, this chapter focuses in detail on concepts of neurotransmission and functions of major neurotransmitters. In primary care, a clinician can expect to spend a significant portion of each day interacting with patients who are trying to manage anxiety, depression, pain, or substance use disorders. Antidepressants, anxiolytics, analgesics, and nonpharmacologic modalities are widely prescribed. Although our knowledge of exact mechanisms of many neurological and affective disorders is incomplete, this chapter presents current hypotheses, concepts, and vocabulary. Knowledge of the long-term effects of adverse childhood experiences and other contributing factors can help clinicians explain the biological basis of these disorders to patients experiencing mental health challenges. Having this understanding also helps the clinician to reduce stigma and promote adherence to management approaches.

The section on sensory function and dysfunction emphasizes pain, as this is a major patient complaint. Motor disorders are very common and must be carefully evaluated and described, so they are given expanded coverage in this chapter. The major disorders of brain function in children include epilepsy and developmental delay, as well as headache and concussion. Cerebrovascular structural changes in older adults increase risk of subdural hematomas, and functional losses can accompany cognitive dysfunction as well as neurodegenerative disorders. Neurovascular disorders are also emphasized in the Gerontological Considerations section of this chapter.

\section{MUSCULOSKELETAL SYSTEM (CHAPTER 16)}

The musculoskeletal system is responsible for the structure of the body and the ability to have purposeful movements. This chapter begins with a review of structure and function of bones, with an emphasis on the remodeling process of dynamic bone maintenance. A discussion of fractures and the fracture healing process follows. The structure and function of joints are described, emphasizing synovial joints and the manifestations of sprains and strains. Particular emphasis is placed on the knee, and the associated ligament and meniscal injuries. This chapter also discusses other common structural and functional disorders, such as herniated disc disease and cumulative trauma disorders. Developmental disorders and sports injuries in children, as well as genetic conditions of muscles and bones, are the focus of the Pediatric Considerations section. Disorders in older adults include general principles of sarcopenia and frailty, as well as osteoporosis, osteoarthritis, and Parkinson-associated Pisa syndrome.

\section{ENDOCRINE SYSTEM (CHAPTER 17)}

The book concludes with the longest chapter, focusing on the endocrine system. This is the third integrative 
system of the body, along with the nervous and immune systems. Found in many locations throughout the body, endocrine glands and cells secrete hormones that act locally and also travel through the circulation to alter the activities of target cells in other organs. General concepts of endocrine signaling are described first, focusing on cellular mechanisms of hormone action, hormone control axes, feedback mechanisms, and temporal regulation of hormone secretion. An important principle of endocrine pathophysiology is that the most common sources of dysfunction fall into three major categories: pathological increases of hormone levels (which are often produced by hormonesecreting tumor cells), pathological decreases of hormone levels (which can be caused by autoimmune destruction of hormone-secreting cells), and insensitivity of target tissues to hormone actions (some of which have genetic causes).

Following the introduction, this chapter content is divided into six major sections that group key content blocks. The initial section provides an overview of the endocrine system. Following this overview begins a more in-depth look at the various parts of the endocrine system. The section covering hypothalamus and pituitary glands provides an in-depth overview of these structures-as sources of direct-acting hormones (oxytocin, vasopressin, growth hormone, and prolactin) and master regulators of other endocrine glands and tissues. The focus then turns to those glands, with separate sections covering adrenals, thyroid, and female and male gonads. The final section of this chapter opens with a review of metabolic physiology that prepares students for detailed coverage of pancreatic hormones and diabetes mellitus. This section begins with an extensive discussion of the hormone insulin, including its mechanisms of signaling and cellular actions, followed by descriptions of the hormones that oppose the actions of insulin. Included are extensive discussions of type 1 and type 2 diabetes, as well as other types of diabetes and related conditions of dysregulated metabolism. Reflecting the unique organization of this chapter, Pediatric and Gerontological Considerations and Key Points appear at the end of each main section rather than at the end of this chapter. Bridge to Clinical practice segments appear in the sections on the thyroid gland and metabolism and diabetes, as management of these disorders is common in primary care settings.

\section{CONNECTING THE DOTS}

As this content overview has emphasized, the conceptually driven organization of this book, linking principles with their clinical applications, aims to reinforce the unique aspects of each organ or system's function and the ways in which alterations of these functions contribute to disease. The rationale for this approach was derived from clinical practice and refined through teaching pathophysiology courses at an advanced level for over 25 years. Having mastered the concepts and vocabulary of function for each system in this way, the reader will best be prepared for later or concurrent courses in pharmacology, clinical diagnosis, and management.

The ability to connect the dots between pathophysiological principles and clinical applications is a major emphasis in this book. This knowledge can provide a rational basis for clinical decision-making and can make it easier to generalize knowledge to new pathological conditions encountered in practice. Knowledge of these core principles assists in learning the strategies and medications used to manage disease states, ultimately facilitating safe and effective advanced patient care.

\section{PATHOPHYSIOLOGY AND THE INDEPENDENT CLINICIAN}

The ultimate goal of graduate education in healthcare practice is to prepare to deliver the highest and safest care possible. This entails knowing not only what signs and symptoms are associated with a certain disorder but why they are associated. Knowing about compensatory mechanisms for impaired oxygen delivery, for example, helps the clinician immediately recognize that a patient presenting with dyspnea and tachypnea is likely hypoxemic, even before applying a pulse oximeter or listening to the lungs. Knowing about the oxygen-carrying capacity of hemoglobin helps the clinician put iron-deficiency anemia at the top of the differential diagnosis list when a patient presents with fatigue, dyspnea on exertion, and pallor.

Knowledge of pathophysiology also directs the logical choice of diagnostic tests. This is something that all novice clinicians struggle with. Which blood test(s) should be ordered? Does this patient need imaging and, if so, which type of imaging will yield the most diagnostically relevant information? One may know to order a complete blood count for suspected anemia, but what other tests will be informative and narrow the differential diagnosis? When the results are obtained, how should they be interpreted? The answers to many of these questions are clearer when there is a strong grounding in pathophysiology. Likewise, the knowledge of normal and abnormal physiology is essential for understanding which medications are the logical choices and why - and equally if not more importantly, why certain medications should not be used in people with certain disease states.

Knowledge of core principles emphasized in this book will make it easier to generalize knowledge to 
new pathological conditions encountered in practice. Novice clinicians should anticipate that they cannot possibly graduate with knowledge of every disease and disorder. There will be times when their patients are diagnosed - usually by a specialist—with a disease they have never heard of or one that was only briefly touched on in their training. A strong foundation in pathophysiology makes it possible to read an online update or journal article about a disease, to understand what normal function is altered, and by extension, how the abnormal state is treated.

Finally, patients and their families expect clinicians to be able to explain and interpret for them what is going on in their particular disease state. Understanding the pathophysiology of a disease is essential to being able to explain the disease in ways your patients will understand. It also helps patients own their disease. With knowledge of what is going on and why certain tests are ordered or why certain medications are prescribed, patients are more likely to take responsibility for those aspects of their illness that they can influence. A clinician can order many tests and prescribe many medications, but ultimately the patient must go get the tests and take the medications. The more successful the clinician is in patient teaching, the greater the likelihood that the patient will feel a partnership with the clinician in the journey to wellness. A deep understanding of pathophysiology facilitates clear explanations tailored to the patient's level of understanding.

\section{REFERENCES}

1. Population Reference Bureau. Fact sheet, aging in the United States. https://www.prb.org/aging-unitedstatesfact-sheet. Published July 15, 2019. Accessed August 26, 2019.

2. World Health Organization. Fact sheet: Ageing and health. https://www.who.int/news-room/fact-sheets/detail/ageingand-health. Published February 5, 2018. Accessed August 26, 2019. 